\title{
A NECESSARY CONDITION IN THE CALCULUS OF VARIATIONS
}

\author{
EDWARD SILVERMAN ${ }^{1}$
}

\begin{abstract}
A necessary condition that an extremal be a relative minimum is derived. The condition includes and may be stronger than the Legendre-Hadamard condition.
\end{abstract}

By considering a slightly more general variation than Hadamard we will obtain a necessary condition in the calculus of variations which may be stronger than the Legendre-Hadamard condition [1, p. 253] and [2, p. 11].

If $\phi \in L(E, F)$ and $p \in E$ let $[p, \phi]=\phi(p)$ where $E$ and $F$ are vector spaces. If $g \in L(E, L(E, F))$ and if $h, k \in E$ let $g h k=[k,[h, g]]$ and $g h^{(2)}=$ ghh.

Let $e_{\alpha} \in R^{v}, \alpha=1, \cdots, v$, and $E_{i} \in R^{N}, i=1, \cdots, N$, be defined by

$$
e_{\alpha}=\left(\delta_{\alpha}^{1}, \cdots, \delta_{\alpha}^{v}\right) \text { and } E_{i}=\left(\delta_{i}^{1}, \cdots, \delta_{i}^{\mathrm{v}}\right) \text {. }
$$

Let $e^{\beta} \in L\left(R^{v}, R\right)=R_{v}$, be defined by $e^{\beta} e_{\alpha}=\delta_{\alpha}^{\beta}$. Let $e^{\beta} E_{i}$ be that element of $L\left(R^{v}, R^{\lambda}\right)$ defined by $\left(e^{\beta} E_{i}\right) e_{\alpha}=\delta_{\alpha}^{\beta} E_{i}$. If $\lambda \in R_{v}$ and $\xi \in R^{N}$ then $(\lambda \xi) e_{\alpha}$ $=\left(\lambda_{\beta} e^{\beta} \xi^{i} E_{i}\right) e_{\alpha}=\lambda_{\alpha} \xi^{i} E_{i}$ (tensor convention for summation). If $\phi \in$ $C^{\prime \prime}\left(L\left(R^{v}, R^{N}\right), F\right)$ we write $\phi_{i}^{\alpha}$ for $\phi^{\prime} e^{\alpha} E_{i}$ and $\phi_{i j}^{\alpha \beta}$ for $\phi^{\prime \prime} e^{\alpha} e^{\beta} E_{i} E_{j}$. Thus if $\lambda=\lambda_{\alpha} e^{\alpha} \in R_{v}$ and $\xi=\xi^{i} E_{i} \in R^{N}$,

$$
\phi_{i j}^{\alpha \beta} \lambda_{\alpha} \lambda_{\beta} \xi^{i} \xi^{j}=\phi^{\prime \prime} e^{\alpha} e^{\beta} E_{i} E_{j} \lambda_{\alpha} \lambda_{\beta} \xi^{i} \xi^{j}=\phi^{\prime \prime}(\lambda \xi)^{(2)} .
$$

Let $G$ be a bounded domain in $R^{v}$ and $f \in C^{\prime \prime}\left(G \times R^{N} \times L\left(R^{v}, R^{N}\right), R\right)$. Let $C^{\prime}=C^{\prime}\left(G, R^{\lambda i}\right)$ and if $z \in C^{\prime}$ let $\|z\|=\sup \left\{\|z(x)\|+\left\|z^{\prime}(x)\right\| \mid x \in G\right\}$. If $z \in C^{\prime}$ let $I_{f}(z)=\int_{G} f(x, z(x), p(x)) d x$ where $p=z^{\prime}$. Suppose that $I_{f}(z) \leqq$ $I_{f}(z+\zeta)$ whenever $\zeta \in C^{\prime}$, support $\zeta \subset \subset G$ and $\|\zeta\|$ is small enough. Then $\phi^{\prime \prime}(0) \geqq 0$ where $\phi(\lambda)=I(z+\lambda \zeta)$ whenever $\zeta \in C^{\prime}$ and support $\zeta \subset \subset G$. Thus

$$
\int_{G}\left\{f_{z z}(P) \zeta^{(2)}(x)+2 f_{z p}(P) \zeta(x) \zeta^{\prime}(x)+f_{p p}(P) \zeta^{\prime(2)}(x)\right\} d x \geqq 0
$$

Received by the editors December 15, 1970.

AMS (MOS) subject classifications (1970). Primary 49B20.

Key words and phrases. Legendre-Hadamard necessary condition, quasi-convex.

1 This research was sponsored in part by the National Science Foundation under grant 6075-50-13955.

(c) American Mathematical Society 1973 
where $P=(x, z(x), p(x))$. Let $x_{0} \in G$, let $n$ be so large that

$$
n^{-1}\left(-x_{0}+\text { support } \zeta\right) \subset \subset-x_{0}+G
$$

and let $\zeta_{n}$ be defined on $G$ with support $\zeta_{n} \subset x_{0}+n^{-1}\left(-x_{0}+\right.$ support $\left.\zeta\right)$ by $\zeta_{n}\left(x_{0}+y\right)=\zeta\left(x_{0}+n y\right) n^{-1}$. Evidently support $\zeta_{n} \subset \subset G, \zeta_{n} \in C^{\prime}$, and

$$
\zeta_{n}^{\prime}\left(x_{0}+y\right)=\zeta^{\prime}\left(x_{0}+n y\right) \text {. }
$$

Using the continuity of $f^{\prime \prime}$ and letting $n \rightarrow \infty$ we see that

$$
f_{p p}\left(x_{0}, z\left(x_{0}\right), p\left(x_{0}\right)\right) \int_{G} \zeta^{\prime(2)}(x) d x \geqq 0 .
$$

By approximations we obtain

LEMMA. If $z$ is a weak relative minimum for I then

$$
f_{p p}\left(x_{0}, z\left(x_{0}\right), p\left(x_{0}\right)\right) \int_{G} \zeta^{\prime 2}(x) d x \geqq 0
$$

for all Lipschitzian $\zeta$ with support $\zeta \subset \subset G$.

Now let $\xi_{1}, \cdots, \xi_{v} \in R^{N}$ and $\lambda^{1}, \cdots, \lambda^{v}$ be a linearly independent set in $R_{v}$. Let $y^{\alpha}=\lambda_{\beta}^{\alpha}\left(x^{\beta}-x_{0}^{\beta}\right)$ and $r=\|y\|$. Let $h>0$ be so small that $x \in G$ if $r \leqq h$. If $i=1, \cdots, N$ let $w^{i}$ be defined on $G^{\prime}=\{y \mid x \in G\}$ with support $w^{i}$ contained in $r \leqq h$ by $w^{i}(y)=\left(h^{2}-r^{2}\right) \xi_{y}^{i} y^{\gamma}$. Then

$$
w_{\alpha}^{i}(y)=-2 y^{\alpha} \xi_{y}^{i} y^{\gamma}+\left(h^{2}-r^{2}\right) \xi_{y}^{i} \delta_{\alpha}^{\gamma}
$$

and $w_{\alpha \beta}^{i}(y)=-2 \xi_{\gamma}^{i}\left(\delta_{\alpha \beta} y^{\gamma}+y^{\alpha} \delta_{\beta}^{\gamma}+y^{\beta} \delta_{\alpha}^{\gamma}\right)$ if $r<h$. Let $T_{\alpha \beta}^{\gamma \varepsilon}=\delta_{\alpha \beta} \delta^{\gamma \varepsilon}+\delta_{\alpha}^{\gamma} \delta_{\beta}^{\varepsilon}+\delta_{\alpha}^{\varepsilon} \delta_{\beta}^{\gamma}$ and note that $\int_{r \leqq h}\left(h^{2}-r^{2}\right) y^{\alpha} y^{\beta} d y=Q \delta_{\alpha \beta} h^{\nu+4}$ for some positive constant $Q$. Hence

$$
\begin{aligned}
\int_{r \leqq h} w_{\alpha}^{i}(y) w_{\beta}^{j}(y) d y & =-\int_{\tau \leqq h} w_{\alpha \beta}^{i}(y) w^{j}(y) d y \\
& =2 \xi_{\gamma}^{i} \int_{r \leqq h}\left(h^{2}-r^{2}\right) \xi_{\varepsilon}^{j} y^{\varepsilon}\left(\delta_{\alpha \beta} y^{\gamma}+y^{\alpha} \delta_{\beta}^{\gamma}+y^{\beta} \delta_{\alpha}^{\gamma}\right) d y \\
& =2 Q h^{\nu+4} \xi_{\gamma}^{i} \xi_{\varepsilon}^{j} T_{\alpha \beta}^{\gamma \varepsilon} \text { since } w^{j} \text { vanishes on } r=h .
\end{aligned}
$$

Let $\zeta^{i}(x)=w^{i}(y)$. Then $\zeta$ is Lipschitzian with support $\zeta \subset \subset G$ and $\zeta_{\alpha}^{i}(x)=w_{\beta}^{i}(y) \lambda_{\alpha}^{\beta}$ if $r<h$. Let $\phi(p)=f\left(x_{0}, z\left(x_{0}\right), p\right)$ and $a_{i j}^{\alpha \beta}=\phi_{i j}^{\alpha \beta}\left(p\left(x_{0}\right)\right)$. Then

$$
\begin{aligned}
0 & \leqq a_{i j}^{\alpha \beta} \int_{G} \zeta_{\alpha}^{i}(x) \zeta_{\beta}^{j}(x) d x \\
& =a_{i j}^{\alpha \beta} \lambda_{\alpha}^{\gamma} \lambda_{\beta}^{\varepsilon} \int_{\tau \leqq h} w_{\gamma}^{i}(y) w_{\varepsilon}^{j}(y)|\operatorname{det} \lambda|^{-1} d y \\
& =2 Q h^{\nu+4}|\operatorname{det} \lambda|^{-1} a_{i j}^{\alpha \beta} \lambda_{\alpha}^{\gamma} \lambda_{\beta}^{\varepsilon} \xi_{\rho}^{i} \xi_{\sigma}^{j} T_{y \varepsilon}^{\rho \sigma} .
\end{aligned}
$$


Thus we have

THEOREM. If $z$ is a weak relative minimum for $I$ then

$$
a_{i j}^{\alpha \beta} \lambda_{z}^{\gamma} \lambda_{\beta}^{\varepsilon} \xi_{\rho}^{i} \xi_{\sigma}^{j} T_{\gamma \varepsilon}^{\rho \sigma} \geqq 0
$$

for all $\lambda^{1}, \cdots, \lambda^{v} \in R_{v}$ and $\xi_{1}, \cdots, \xi_{v} \in R^{N}$.

If we set $\lambda^{1}=\lambda, \xi_{1}=\xi$ and $\lambda^{\rho}=0, \xi_{\sigma}=0$ for $\rho \neq 1, \sigma \neq 1$, then we get the Legendre-Hadamard condition

$$
a_{i j}^{\alpha \beta} \lambda_{\alpha} \lambda_{\beta} \xi^{i} \xi^{j} \geqq 0
$$

for all $\lambda \in R_{v}$ and $\xi \in R^{N}$. If $f \in C^{\prime \prime}$ then $f$ is quasi-convex if the LegendreHadamard condition holds [2, p. 112]. Let us say that $f$ is pseudo-convex if $f \in C^{\prime \prime}$ and (*) holds. (In fact, Morrey defined quasi-convexity without imposing differentiability conditions on $f$.) Within the class of $C^{\prime \prime}$ functions it is evident that pseudo-convexity implies quasi-convexity.

Let us say $\left[2\right.$, p. 114] that $f$ is strongly quasi-convex if $f \in C^{\prime \prime}$ and if

$$
\int_{G} f\left(x_{0}, z_{0}, p_{0}+\zeta^{\prime}(x)\right) d x \geqq f\left(x_{0}, z_{0}, p_{0}\right) \cdot m(G)
$$

for any constant $\left(x_{0}, z_{0}, p_{0}\right)$, any bounded domain $G$, and any Lipschitz $\zeta$ with support $\zeta \subset \subset G$. Let $z^{i}(x)=\left(p_{0}\right)_{\alpha}^{i} x^{\alpha}$ so that $z^{\prime}=p_{0}$. Let $F(x, z, p)=$ $f\left(x_{0}, z_{0}, p\right)$. if $f$ is strongly quasi-convex and if $\left\{\zeta_{n}\right\}$ is a sequence of Lipschitz functions with support $\zeta_{n} \subset \subset G$ and $\left\|\zeta_{n}\right\| \rightarrow 0$, then $I_{F}(z) \leqq$ $\lim \inf _{n \rightarrow \infty} I_{F}\left(z+\zeta_{n}\right)$. Thus $F$, and hence $f$, satisfies $\left(^{*}\right)$ so that $f$ is pseudoconvex if $f$ is strongly quasi-convex. Thus to show that there exist quasiconvex functions which are not strongly quasi-convex it is sufficient to show that there exist functions satisfying $\left({ }^{* *}\right)$ but not $\left({ }^{*}\right)$.

\section{REFERENCES}

1. J. Hadamard, Leçons sur la propagation des ondes et les équations de l'hydrodynamique, Hermann, Paris, 1903.

2. C. B. Morrey, Jr., Multiple integrals in the calculus of variations, Die Grundlehren der math. Wissenschaften, Band 130, Springer-Verlag, New York, 1966. MR 34 \#2380.

Department of Mathematics, Purdue University, West Lafayette, Indiana 47907 